\title{
OBSERVATIONS ON THE NORMAL ANATOMY OF THE BRONCHIAL ARTERIES
}

\author{
BY \\ L. CUDKOWICZ* AND J. B. ARMSTRONG \\ From the Department of Medicine, Postgraduate Medical School of London
}

(RECEIVED FOR PUBLICATION OCTOBER 16, 1951)

The existence of systemic arteries to the lungs has been known for many centuries. Ruysch (1732) claimed to be the first to have described the bronchial arteries, but Galen had already done so in the second century A.D.

During the nineteenth century the anatomy of the bronchial arteries was studied in detail and their possible function considered. Reisseisen and von Soemmering (1808) defined the bronchial arteries as "vasa nutritiva" of the lungs, and showed that they accompanied the bronchi. Le Fort (1858) showed that the bronchial arteries continue along the bronchial tree as far as the respiratory bronchioli. Von Luschka (1863) drew attention to the ramification of the bronchial arterioles in the bronchial mucosa and suggested that the capillaries of the bronchial arterioles communicated near the alveolar ducts with those derived from the pulmonary arteries.

Virchow (1856) showed that occlusion of a lobar pulmonary artery branch did not result in necrosis of the lobe. He concluded therefore that the viability of the lobe.did not depend on the pulmonary artery, but was maintained by the bronchial arteries. Rindfleisch (1878) considered the bronchial arteries to be end arteries. Küttner (1878), by dye injections in rabbit lungs, showed that the terminal branches of the pulmonary arteries were nowhere in communication with vessels of a similar calibre, and concluded that communication between the pulmonary artery bed and the bronchial arteries, if it existed, must be capillary only. Zuckerkandl (1881 and 1883) demonstrated that the bronchial arteries supplied the visceral pleura in man, and thought that the capillaries of the pulmonary and bronchial arteries were in communication.

Miller (1947), at the beginning of this century, described the course of the bronchial arteries. He showed that they ran from the aorta to the main bronchi and followed these distally to the hila. There they divided and continued in the walls of the bronchi as far as the alveolar ducts. From the fibrous tunic, in which an arterial plexus was formed, small branches penetrated the muscle layer to reach the tunica propria of the bronchus and then continued to run in the long axis of the bronchus.

Miller did not think that the problem of anastomosis between the two circulations had been adequately studied by previous workers, and contended that the use of a

* In receipt of a grant from the Medical Research Council. 
fluid injection medium capable of traversing the capillary beds led to erroneous observations. With the aid of a viscid injection mass he showed that communications existed only between the venous capillaries of the two systems. The capillaries from the bronchial arteries constituted a source for the pulmonary veins and joined the pulmonary venules as additional venous radicles. Hence the pulmonary veins conveyed a proportion of systemic venous blood to the left auricle. $\mathrm{He}$ injected Berlin blue in gelatin at a pressure of $110 \mathrm{~mm}$. of mercury into the vessels of the dog's lungs and found that on injecting $(a)$ the pulmonary artery, leaving the pulmonary veins patent, the bronchial arteries did not fill ; $(b)$ the pulmonary veins, with the pulmonary artery patent, there was slight filling of the bronchial arteries; (c) the pulmonary artery with the pulmonary veins clamped, there was slight filling of the bronchial arteries; $(d)$ the pulmonary veins with the pulmonary artery clamped, the bronchial arteries filled completely. In addition (e) the pulmonary veins were injected alone until a free flow issued from the pulmonary artery. The pulmonary veins were then clamped and a solution of gelatin containing vermilion granules was forced into the pulmonary artery. The pulmonary veins and the bronchial arteries remained blue. The pulmonary artery became red.

Miller concluded from these studies that the injection of the bronchial arteries via the pulmonary arteries becomes possible only if the pulmonary veins are occluded, that is, as a result of back pressure along those venous radicles which participate in the formation of the pulmonary veins. Karsner and Ash (1912) showed that as long as a definite pressure was maintained in both the pulmonary artery bed and the bronchial arterial circuit little admixture of the injection media would take place. Reduction of the pressure in one system to zero might bring about penetration by the opposite system. They also demonstrated that experimental emboli did not produce necrosis of the affected lung segments as long as the bronchial arteries were patent.

Berry, Brailsford, and Daly (1931) and Daly (1935-6) confirmed Miller's observation and also found that the bronchial arteries supplied both vasa vasorum to the pulmonary arteries and branches to all pulmonary nerves and to the lymphatic structures in the lungs, the hila, and at the carina. Mathes, Holman, and Reichert $(1930,1932)$ used a radio-opaque injection mass to study the bronchial arteries in the lungs of a dog, and reached the same conclusion as Miller. They agreed that the pulmonary arteries were end arteries and did not communicate with vessels of similar calibre derived from the bronchial arteries. Berry (1935) injected the lungs of stillborn infants with $40 \%$ barium sulphate in $2.5 \%$ gelatin and confirmed the findings of Miller.

The normal anatomy of the bronchial arteries appears therefore well established, in particular their mode of distribution to the bronchi and the ancillary distribution to other lung structures.

In the present study the anatomy of the bronchial arteries is examined once more in normal human post-mortem material, using a new technique. Particular attention has been paid to the intra-pulmonary distribution of the bronchial arteries in normal lungs as a necessary preliminary to later studies of the changes in the bronchial arteries found in disease. 


\section{MATERIAL}

The lungs examined in this series were normal in structure and showed no evidence of histological lesions in the lung vessels, the alveoli, or bronchial tree, and the radiographic appearances of the bronchial arteries were, therefore, assumed to show normal configurations. In 10 preparations the vascular patterns, obtained with the aid of a radio-opaque injection mass, showed uniformity and similarity of detail. It is therefore thought reasonable to use these 10 studies as criteria of normality. Histological sections have been taken of all injected lungs and were studied in conjunction with the radiological observations. Details of the cases from which the material was obtained are shown in Table I.

TABLE I

Causes of Death in Patients whose Lungs were Investigated

\begin{tabular}{|c|c|c|c|c|}
\hline Case & Age & Sex & Diagnosis at Time of Death & Lungs Injected \\
\hline $\begin{array}{l}1 \\
2 \\
3 \\
4 \\
5 \\
6\end{array}$ & $\begin{array}{l}61 \\
45 \\
49 \\
43 \\
70 \\
46\end{array}$ & $\begin{array}{l}\text { M. } \\
\text { M. } \\
\text { M. } \\
\text { M. } \\
\text { F. } \\
\text { M. }\end{array}$ & $\begin{array}{l}\text { Chronic leukaemia } \\
\text { Chronic nephritis } \\
\text { Coronary thrombosis } \\
\text { Cerebral glioma } \\
\text { Carcinoma of cervix } \\
\text { Tabetic vesical crisis (sudden death after }\end{array}$ & $\begin{array}{l}R . \text { and } L \text {. } \\
R \text {. and } L \text {. } \\
R \text {. and } L \text {. } \\
R \text {. and } L \text {. } \\
R \text {. and } L \text {. }\end{array}$ \\
\hline 7 & 66 & M. & $\begin{array}{l}\text { Post-operatively following partial gas- } \\
\text { trectomy for chronic peptic ulcer }\end{array}$ & $\begin{array}{l}\text { R. and } \mathrm{L} \text {. } \\
\text { R. and } \mathrm{L} \text {. }\end{array}$ \\
\hline 8 & 61 & M. & $\begin{array}{l}\text { Carcinoma of right middle lobe bronchus; } \\
\text { cerebral metastases }\end{array}$ & L. lung normal \\
\hline $\begin{array}{r}9 \\
10\end{array}$ & $\begin{array}{l}57 \\
53\end{array}$ & $\begin{array}{l}\mathrm{F} . \\
\mathrm{M} \text {. }\end{array}$ & $\begin{array}{l}\text { Carcinoma of right upper lobe bronchus } \\
\text { Carcinoma of right lower lobe bronchus }\end{array}$ & $\begin{array}{l}\text { L. lung normal } \\
\text { L. lung normal }\end{array}$ \\
\hline
\end{tabular}

\section{METHODS}

The bronchial arteries were outlined by a contrast medium injected into the aorta under pressures equal to the mean systolic pressure recorded during life. Radiographs were taken as soon as the injection medium was thought to have cooled and hardened sufficiently to prevent leakage from small vessels during the manipulations of the specimen. Antero-posterior views and lateral views with the hilum facing either the tube or the plate were used. It was found that all the injected arteries were shown equally well in each of these positions.

Injection Medium.-The injection medium was that used by Dr. J. P. Shillingford (1950) at the London Hospital for his work on the coronary circulation. The solution consisted of an equal amount of bismuth oxychloride cream* and $50 \%$ gelatin in water. It flowed evenly at temperatures between $25^{\circ}$ and $35^{\circ} \mathrm{C}$. and filled vessels of $60 \mu$ in diameter and above. At no time did penetration into the capillary bed obscure the vascular relationships.

* The bismuth oxychloride cream was prepared by adding $1,000 \mathrm{ml}$. of concentrated hydrochloric acid to $515 \mathrm{~g}$. of bismuth oxycarbonate; $1,300 \mathrm{ml}$. of distilled water were added to the solution and the whole filtered into approximately 30 litres of London tap water. The filtration into the tap water precipitated the bismuth oxychloride cream and rendered the solution neutral. After the precipitate had settled the clear neutral supernatant fluid was decanted. The settled wet precipitate of the bismuth oxychloride cream was stirred well, and $250 \mathrm{ml}$. of this were mixed with an equal volume of $50 \%$ gelatin in water before use. 
Apparatus.-The apparatus used throughout this work is shown in Fig. X. A 6-litre bottle with a low-set outflow was used as a pressure reservoir. A stopcock was incorporated in one of the two glass tubes in the cork, through which air was pumped by an attached sphygmomanometer bulb. A length of pressure tubing connected the reservoir with a glass tube in the cork of a $500 \mathrm{ml}$. flask containing the injection medium. A second length of tubing allowed interruption of the flow of the medium without losing the head of pressure in the reservoir, the volume of which was sufficient to maintain the pressure for some minutes when the injection medium was flowing. A mercury manometer was incorporated in the circuit between the reservoir and the flask.

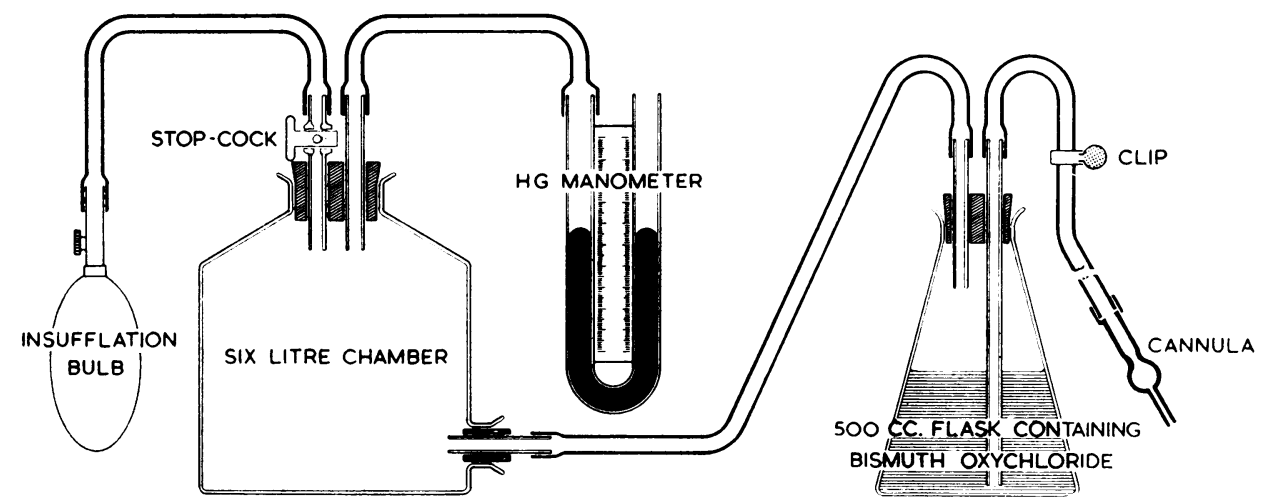

Fig. X.-Diagram of the apparatus.

\section{TECHNIQUE}

In five cases injections were attempted with the lungs in situ. The left common carotid artery was cannulated, the aorta was tied off above the heart and diaphragm and tourniquets were applied to the arms. The medium was injected at $40^{\circ} \mathrm{C}$. after flushing the thoracic aorta with warm saline. In all five cases the bronchial arteries failed to fill, possibly because the medium in the aorta cooled rapidly and solidified.

In the technique finally adopted the pericardium was incised and the heart was removed. The posterior pericardial membrane was opened to expose the oesophagus and the descending aorta. Both structures were divided just above the diaphragm and the aorta at that level was clamped. The lungs, one at a time, were then gently everted across the midline to expose the paravertebral gutter. The parietal pleura in that position and about two inches away from the vertebral bodies was incised longitudinally from the first rib down as far as the diaphragm. The intercostal arteries were thereby severed and their proximal stumps, with the overlying pleura, were reflected medially across the anterior longitudinal ligaments. The same procedure was carried out on the opposite side. Thus, the whole mediastinum was freed of its posterior attachments. The trachea was cut across opposite the first rib and the distal portion was clamped. The subclavian arteries were severed at the same level and the proximal ends ligated. The common carotid arteries were divided and the thoracic contents, less the heart, removed in bulk. The right common carotid artery and all free stumps of the intercostal arteries, which could be easily identified in the free edge of the parietal pleura, were securely ligated. The whole preparation was suspended in a warm water bath at a temperature of $40^{\circ} \mathrm{C}$. The left common carotid artery was cannulated and warm saline was injected through the cannula at the systolic pressure of the patient in life. All other intercostal stumps which 
had not already been secured were now easily tied off as the saline usually spurted powerfully out of the patent stumps.

The preparation was leak-proof, and as more saline entered the aorta under constant pressure the lungs began to swell. After some minutes blood-stained saline issued from the pulmonary veins. This flow was maintained until it became constant on both sides. Approximately 6 litres of saline were needed before this state was reached, and it took about 25 minutes. The bismuth oxychloride/gelatin cream was then substituted for the saline and was allowed to flow for another 30 minutes at the same pressure. About $400 \mathrm{ml}$. of bismuth cream usually entered the preparation in that period. It never appeared in the pulmonary veins or arteries. The lungs were then cooled and radiographs taken.

In some cases the relationship of the bronchial arteries to the bronchi was further defined by injecting the bronchial tree directly with the bismuth/gelatin cream.

\section{RESULTS}

Origins of Bronchial Arteries.-The radiographic studies of these 10 preparations suggest that the bronchial arteries emerge in varying numbers, often two to each lung, from the inferior aspect of the arch and the descending aorta, near the level of the fifth and sixth dorsal vertebrae. These findings were not constant and as many as three vessels were noted to enter the hilum on one or other side. Origins from sources other than the aorta were not noted in the present 10 cases.* Some variation in the size of the vessels was very common.

Extrapulmonary Course.-Before reaching the hila the bronchial arteries ran laterally from the aorta and crossed the oesophagus anteriorly or posteriorly. The left branches appeared to cross behind the oesophagus fairly frequently. Branches emerging from the undersurface of the arch of the aorta constituted true anterior bronchial arteries in virtue of their course anteriorly across the main bronchi and immediately lateral to the bifurcation of the trachea. In their path from the aorta to the hilum the bronchial arteries sent branches to the mediastinum, oesophagus, hilar lymph nodes, and the vagi. On reaching the main bronchus on each side, they formed an annulus surrounding the bronchus, and it was from this structure that the true bronchial and pleural arteries emerged.

Visceral Pleural Arteries.-The medial pleura received vessels directly from the annulus. The anterior, lateral, and interlobar visceral pleura appeared to receive arteries from branches of the bronchial arteries within the lung substance. These vessels, which could be seen with the naked eye, appeared over the lateral pleura after emerging from the underlying lung. Radiographically they could be distinguished from the bronchial arteries by their superficial position, which was particularly obvious in lateral views, their independent course from the bronchial tree, and their relatively larger calibre at peripheral sites where the true bronchial vessels usually disappeared.

We suggest that branches in the medial visceral pleura, which radiate from the hilum directly, should be referred to as " hilar pleural branches," and the vessels not arising from the annulus at the hilum as "lateral pleural branches." Those at the

* Although the intercostal arteries were divided about 2 in. away from their aortic origin, subsequent dissection and removal of the lungs revealed no evidence of systemic arteries to the lungs which might have arisen from the distal intercostal arteries. 
diaphragmatic surface may be called "basal pleural branches" and the vessels in the fissures "right" or "left oblique fissure branches" and "right horizontal fissure branches" respectively.

On many occasions we observed one branch of the hilar pleural vessels extending to the apical pleura, particularly where apical lesions apparently led to an increase in its size. We suggest that this branch might be called the "apical pleural branch" (Fig. 1). The other pleural branches were less constant and it is therefore probably simpler to retain for these the non-specific nomenclature already referred to.

True Bronchial Arteries.-The systemic arteries to the lungs entered the hilum to form a communicating arc round the main bronchi from which the bronchial arterial divisions radiated along the major bronchi. They adhered closely to the bronchial tree and appeared to follow the same course (Figs. 1 and 2). They bifurcated with the bronchi and sent two divisions along each bronchus, one on each side of its wall, which often tended to form an intercommunicating network in the fibrous coat of the bronchus. Smaller twigs penetrated the bronchial walls and appeared as a similar network in the submucosa. In the present 10 normal cases no pre-capillary anastomoses could be demonstrated between the bronchial and pulmonary arteries.

By the arrangement outlined the bronchial arteries tended on the radiograph to outline the position of the bronchial tree (Fig. 3). In view of this constant pattern of the bronchial arteries in normal lungs it might be convenient to use the accepted nomenclature of the bronchial tree (Thoracic Society, 1950) for the main bronchial arterial divisions (Fig. 4). The arterial divisions as seen in a left and a right lateral view are outlined by Figs. 5 and 6 respectively. This nomenclature should be applied to the set of vessels accompanying the respective bronchus. A finer division of nomenclature would not serve a useful purpose. The vascular configurations can be identified with ease in normal lungs as the general pattern remains constant. No tracings of the pleural divisions have been made, for reasons already indicated, but the constancy of the apical pleural branch is thought to merit emphasis once more.

Histological Studies of the Normal Bronchial Arteries

For the purpose of histological study the injected lungs were inflated via the trachea with $10 \%$ formol saline at a pressure of about $25 \mathrm{~mm}$. of mercury and suspended in the same solution for 48 hours. Blocks were, as a rule, taken from (a) hilar lymph nodes, (b) vagus nerve near the hilum, (c) pulmonary artery at the hilum, $(d)$ main bronchi, (e) broncho-vascular bundles at random and about three inches from hilum, $(f)$ peripheral visceral pleura, including the apex, $(g)$ areas of interest as revealed by radiographs, and (h) random lung sections.

The sections were stained routinely with haematoxylin and eosin. The bismuth-filled arteries fixed well and the bismuth cream adhered firmly to the vessel walls and remained in the lumen as a homogeneous white mass. Under the microscope the mass appeared as coarse black granules in the vessel lumen and made the identification of the bronchial arteries in the lung substance easy. The pulmonary artery and pulmonary veins remained, of course, uninjected and showed no evidence of the bismuth granules in their lumina.

\section{RESULTS}

Lymph Nodes.-In all instances bismuth-filled arteries were seen in the capsule and stroma of lymph nodes. The vessels in the stroma were smaller than the capsular vessels and tended to run in the septa separating the lymph follicles. The calibre of 


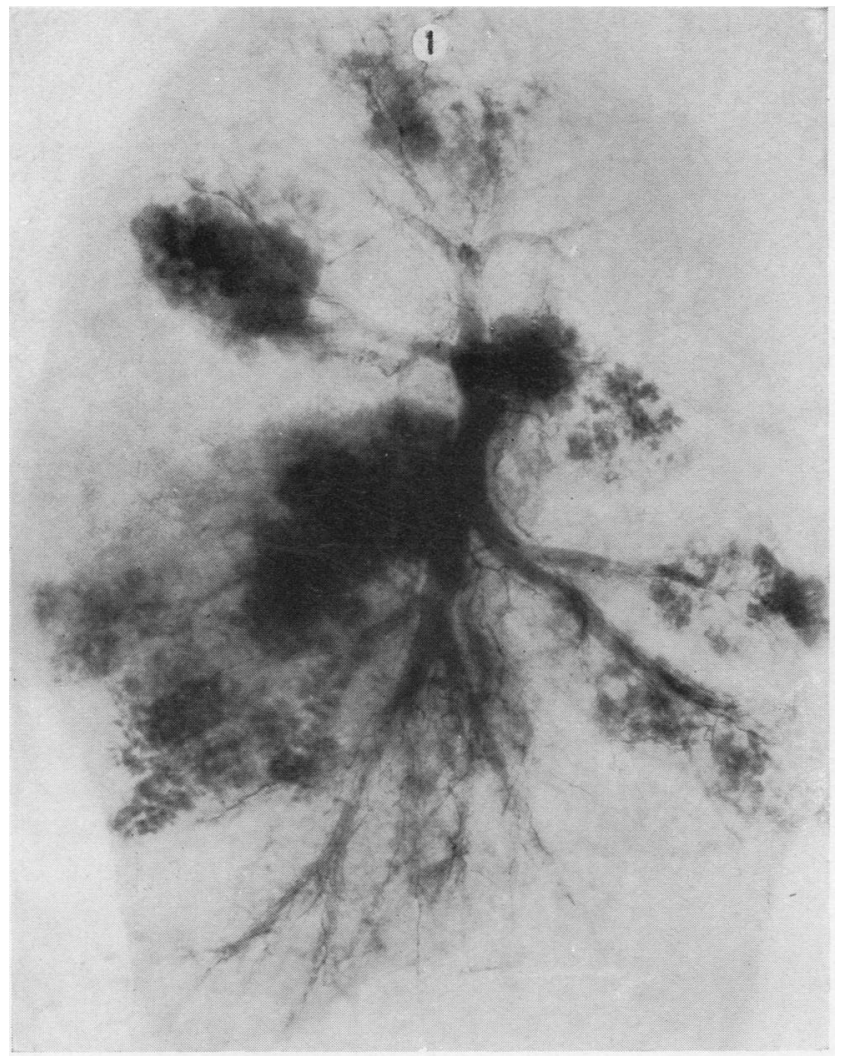

Fig. 1.-I ateral view (Case 3) of right bronchial tree superimposed on right bronchial arterial pattern.

$1=$ Apical pleural branch. Some alveolar fogging has occurred. The hilum faces away from $x$-ray tube. About one-third of normal size.

Fig. 1

Fig. 2.-Lateral view (Case 4) of left bronchial tree superimposed on left bronchial arterial pattern. The relationship of the bronchial arteries to the bronchi, visceral pleura, and lymph node at the left hilum can be seen.

$1=$ apical pleural branches; $2=$ interlobar septal branches; $3=$ branch to hilar lymph node; $4=$ bronchial arteries accompanying the inferior lingular bronchus.

The hilum faces away from $x$-ray tube. About one-third of normal size.

Figs. 1 and 2 are radiographs of preparations in which the bronchial arteries were injected as described in the text, and the bronchi subsequently filled with a radio-opaque medium.

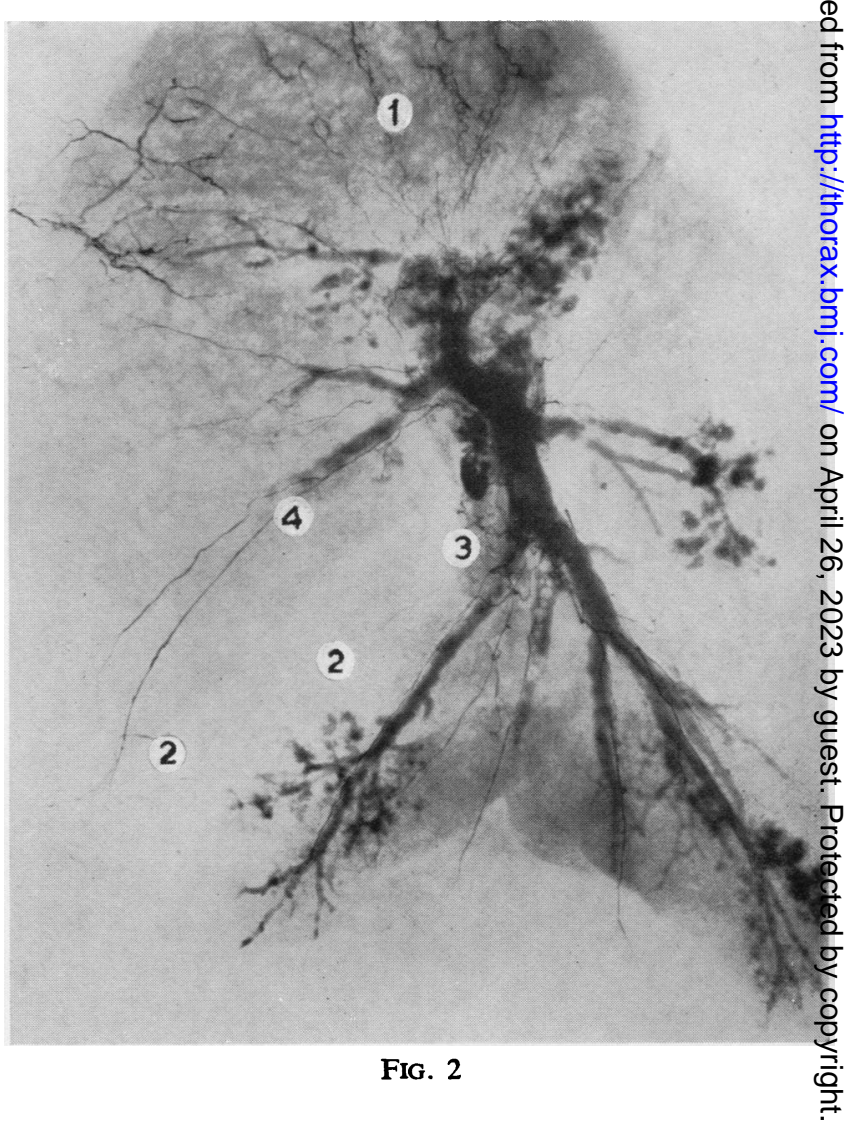


FIG. 3.-The distribution of the bronchial arteries as seen in an anteroposterior view of a right lung (Case 5). The bronchial tree becomes silhouetted in this view by the bronchial arteries.

FiG. 4.-Radiograph of right and left lungs (Case 1) with trachea and aorta attached to show origin of bronchial arteries from aorta and their distribution in the lungs as seen in an antero-posterior view. (Distortion of vessels at hila is due to ligature around main bronchi.)

Loft upper lob': A apical branch; $\mathbf{B}=$ anterior branch; $C$ superior lingular branch; $D=$ inferior lingular branch; $\mathrm{E}=$ posterior branch.

Loft lower lob,: $\mathbf{F}$ lateral branch: $\mathbf{G}=$ anterior branch; $\mathbf{H}$-posterior branch I - apical branch.

Right uppor lob? : $\mathrm{K}=$ pleural branch ; $\mathbf{L}=$ apical branch: $\mathbf{M}$ - anterior branch [Posterior branch not well seen in this preparation.]

Kight middle lobe: $\mathrm{N}$ lateral branch; $\mathrm{O}$ medial branch; $\mathbf{P}=$ interlobar branch (pleural).

Right lower lob": $\mathbf{Q}=$ lateral branch; $\mathbf{R} \cdots$ anterior branch; $\mathbf{S}=$ cardiac (medial) branch; $\mathrm{T}$ posterior branch. [Apical branch not well seen in this preparation ]

FIG. 3

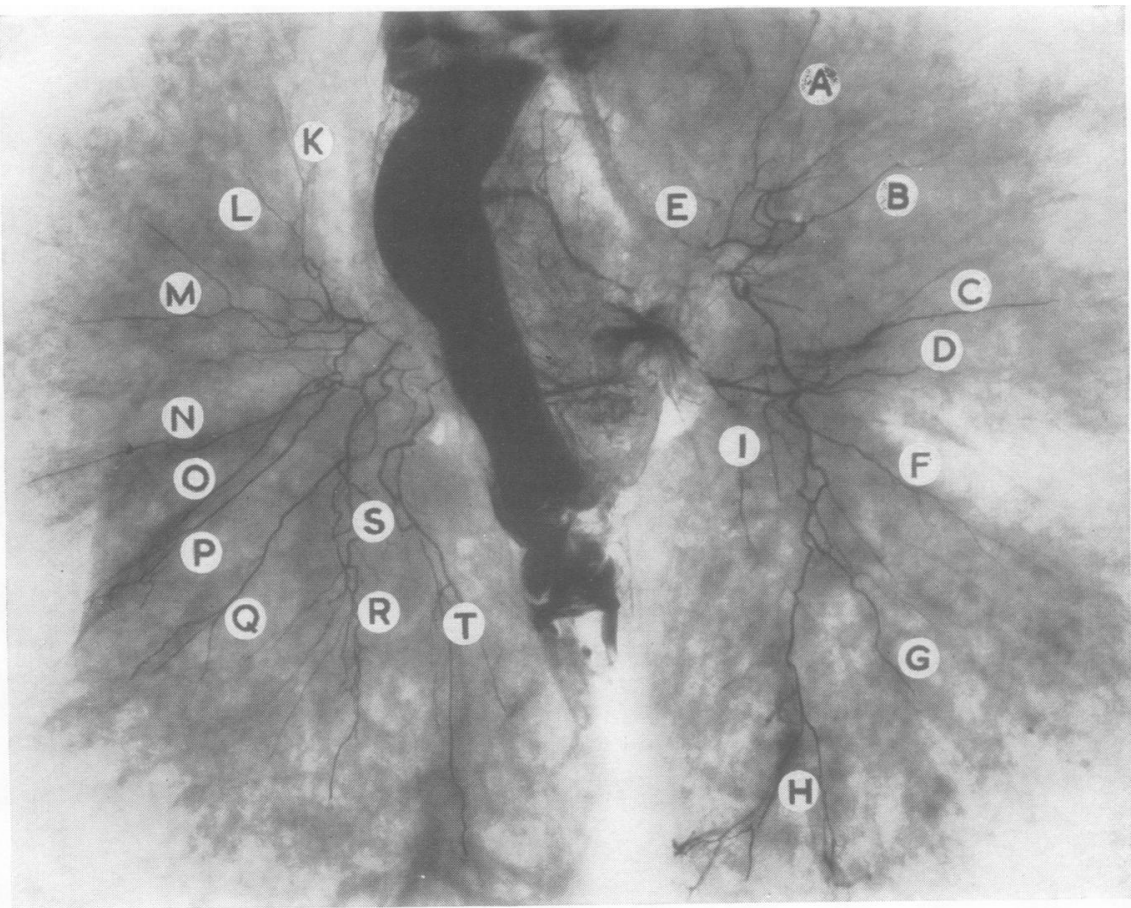

FIG. 4 
FIG. 5.-Standard pattern of left bronchial arteries seen in a lateral view of a left lung (Case 6). (Hilum faces $x$-ray tube.)

Upper lob?: $\mathrm{A}=$ apical pleural branch; $\mathrm{B}=$ posterior branch; $C=$ apical branch; $D=$ anterior branch; $E=$ superior lingular branch; $F=$ inferior lingular branch; $G=$ annulus.

Lower lobe: $\mathbf{H}=$ interlobar pleural branch; $\mathbf{I}=$ anterior branch; $\mathrm{K}=$ lateral branch; $\mathrm{L}=$ posterior branch; $\mathbf{M}=$ apical branch.

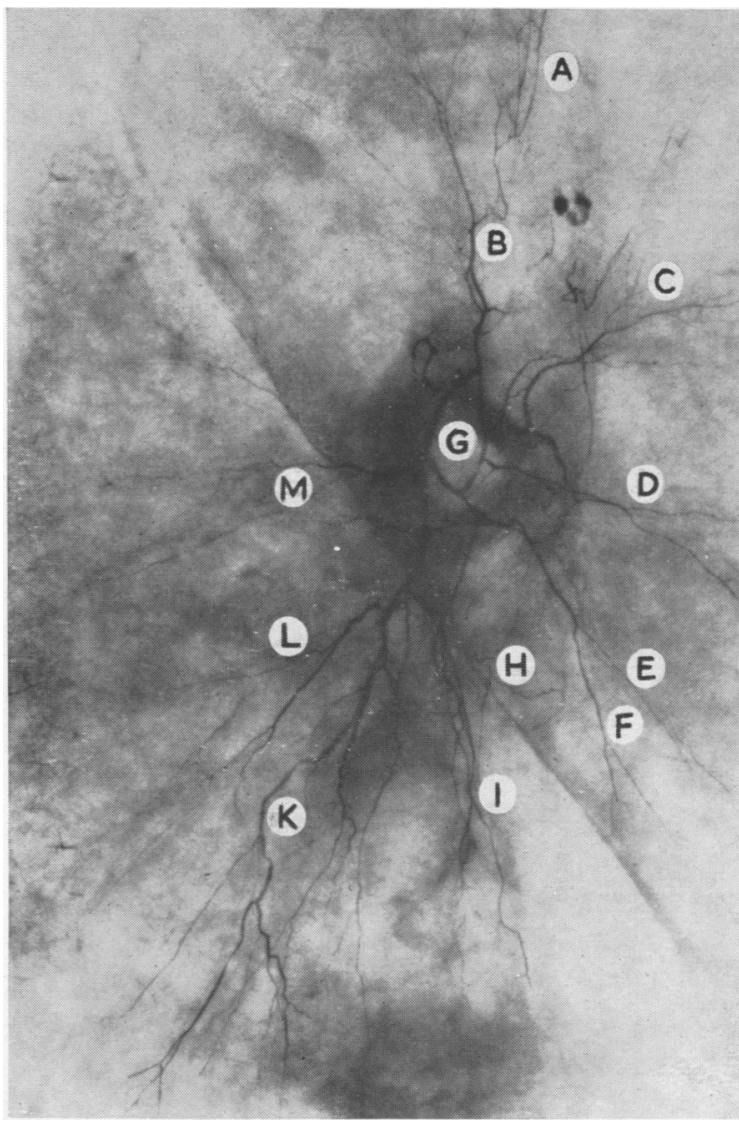

FIG. 5

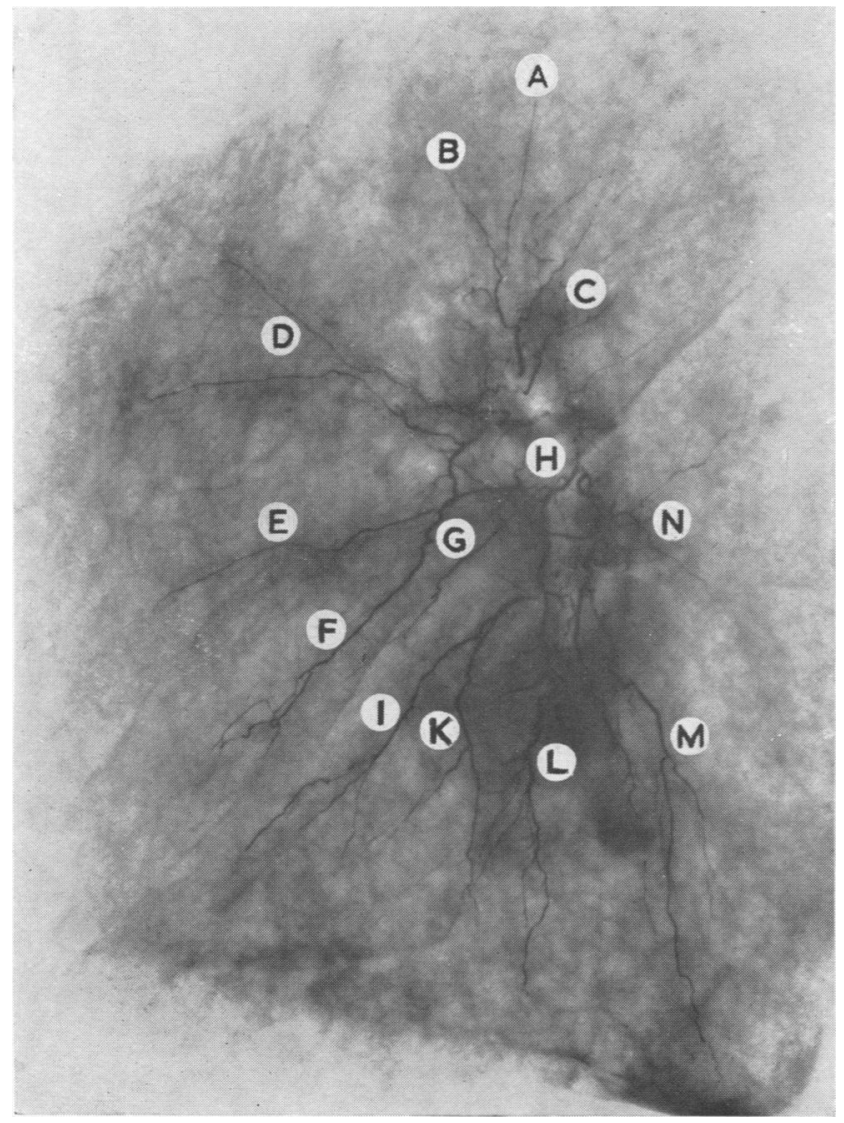

FIG. 6.-Standard pattern of right bronchial arteries seen in a lateral view of a right lung (Case 7). (Hilum faces $x$-ray tube.)

FIG. 6 
the vessels varied directly with the size of the lymph nodes. Lymph nodes situated near the adventitial coat of a major bronchus received their bronchial arterial blood supply from the vessels supplying the adjacent bronchus (Fig. 7).

Pulmonary Artery.-In seven instances the vasa vasorum to the pulmonary arteries could be identified, and were seen to be well filled with bismuth. They were vessels of more than $100 \mu$ in diameter, and seen in the adventitia of the pulmonary artery (Fig. 8).

Vagus.-In five sections of the vagi from the region of the major bronchi wellfilled bronchial arterioles were seen in the perineurium. On two occasions the vessels were seen to penetrate the perineurium and to arrange themselves between the nerve fibres. No uninjected arterioles were visible near the nerve trunks, suggesting therefore that, in the region of the lung hila, at least, the bronchial arteries are nutritive to the pulmonary vago-sympathetic trunks (Fig. 9).

Main Bronchi.-The main bronchi in transverse section usually revealed three to four large bronchial arteries in the peribronchial coat. Branches from there passed through the cartilaginous gaps and, after supplying the perichondrium, entered the tunica propria as small but very obviously injected vessels. Considerable numbers were seen within the glandular elements and adjacent to the basement membrane of the ciliated epithelium (Fig. 10).

Smaller Bronchi.-In the smaller bronchi the bronchial arterial arrangements were the same'as in the larger, but the vessels were naturally of a smaller calibre. The distribution to the structures of bronchial walls was also the same. In the region of the bronchioles small bronchial arterioles were seen within and without the bronchiolar walls. Those which lay outside the wall ran in the fibrous septum between the accompanying pulmonary arteriole and the bronchiole (Fig. 11). The pulmonary veins lay in the alveolar septa a little further away from the bronchiole and were duplicated at this level.

Lung Parenchyma.-Random sections of lung from the periphery in the present 10 cases showed normal anatomical features of the lobules. The capillaries were not distended as a result of the large amounts of saline which had been flushed through the preparation before the injection with bismuth. Well-injected bronchial arterioles were seen in the interlobular septa (Fig. 12). Near the alveolar ducts the smallest bismuth-filled arterioles were seen adjacent to the mucosa. Their diameter was probably just over $80 \mu$. The corresponding pulmonary arterioles and venules were larger and free from bismuth. Larger, well-filled bronchial arterioles were, however, visible in the supporting framework of the alveolar epithelium (Fig. 13), and they appeared to enter the air sacs with strands of elastic tissue which insinuated themselves between the alveoli as outgrowths of the interlobular septa (Fig. 12).

Pleura.-Sections of the visceral pleura showed that the systemic arteries lay within the subserosal coat. Occasional vessels penetrated the pleura from the underlying lung. More frequently, and particularly at the apex, the vessels appeared to lie entirely in the pleural membrane, which would accord with the macroscopic observation that the pleural arteries of the medial pleura, and the apical pleural branch, coursed from the hilum superficially. All visible arteries in the pleura of these normal lungs were fully injected, indicating that they were all derived from the bronchial arteries (Fig. 14). 
Fig. 7.-Photomicrograph of a transverse section of a main bronchus with peri-bronchial lymph node (Case 8). Bronchial arteries can be seen in (1) the stroma of hilar lymph node, (2) capsule of node, and (3) near vagal bundle. $\times 7$.

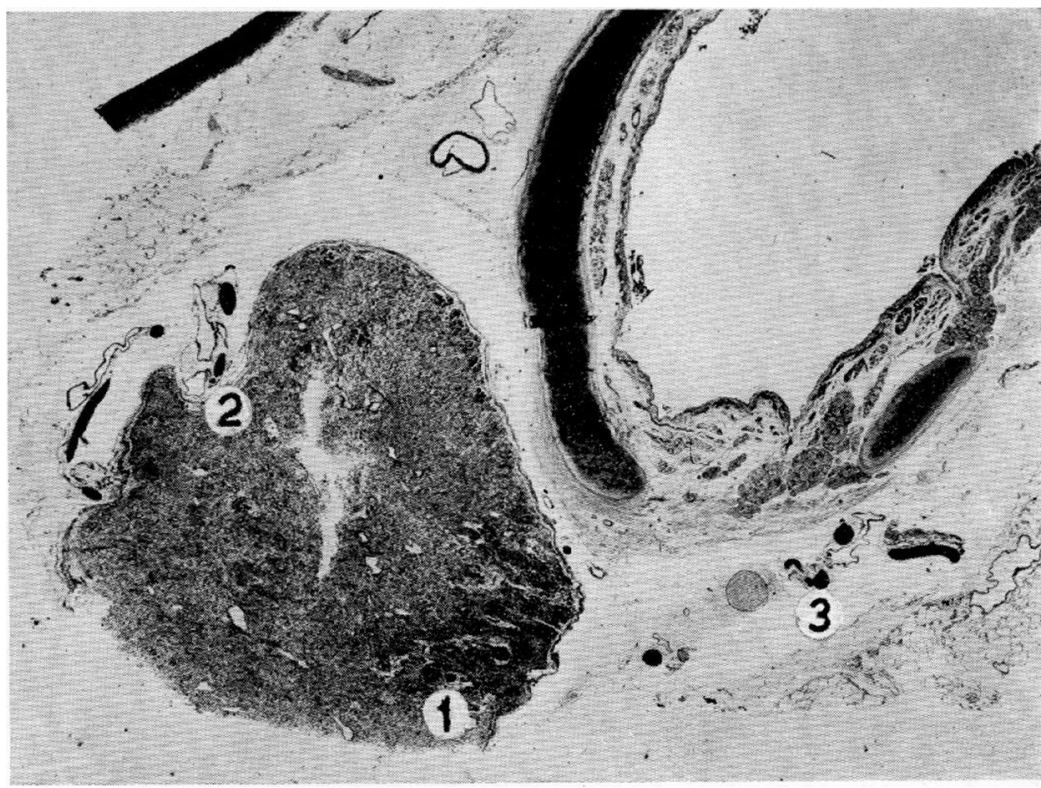

FIG. 7

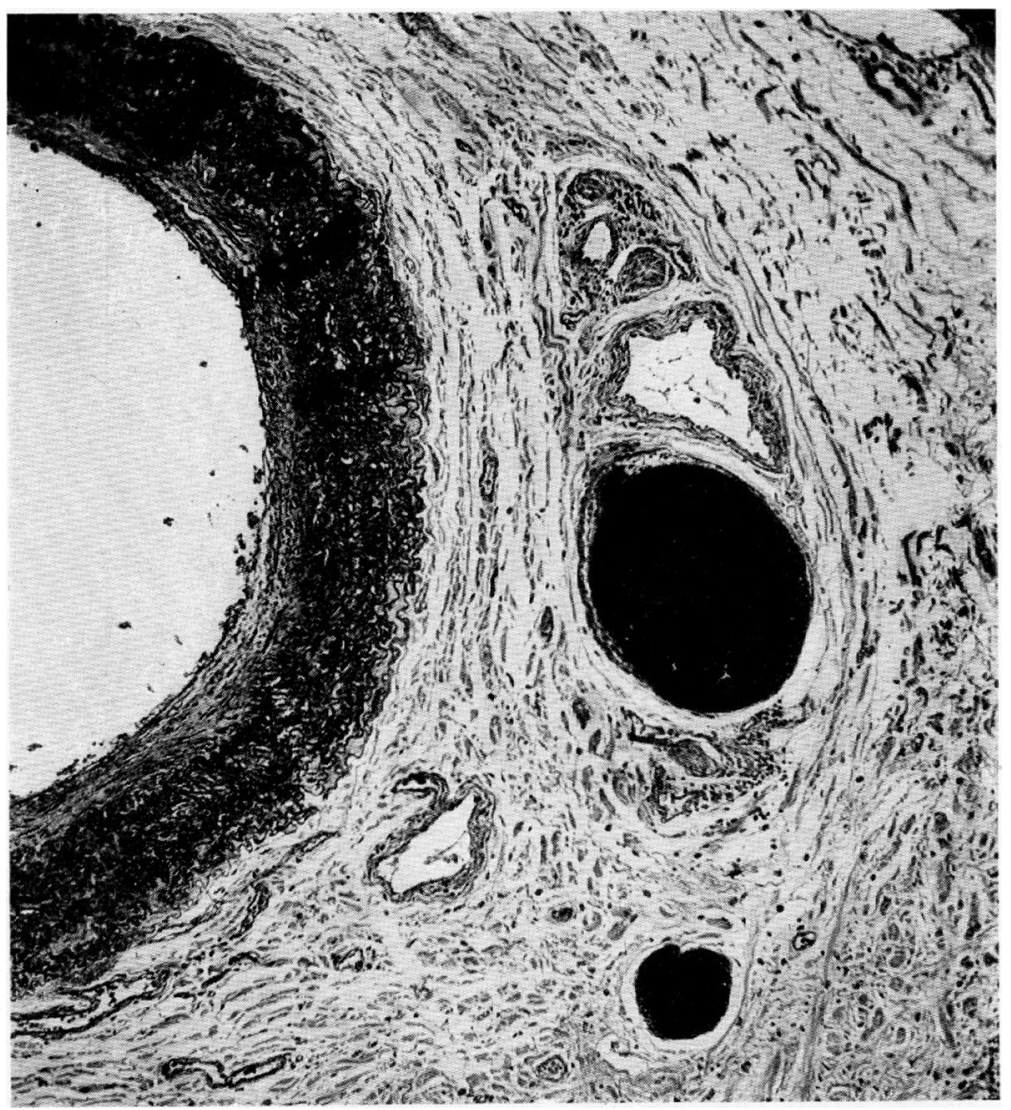

Fig. 8.-Photomicrograph of a pulmon ary artery branch near the hilum 3 (Case 8). Bronchial arterioles of considerable diameter were present as vasa vasorum in the adventitia of the pulmonary artery. $\times 65$.

FIG. 8 
Fig. 9.-Photomicrograph of vagal nerve bundle near the left hilum (Case 10). A bronchial arteriole is visible in the perineurium. $\times 105$
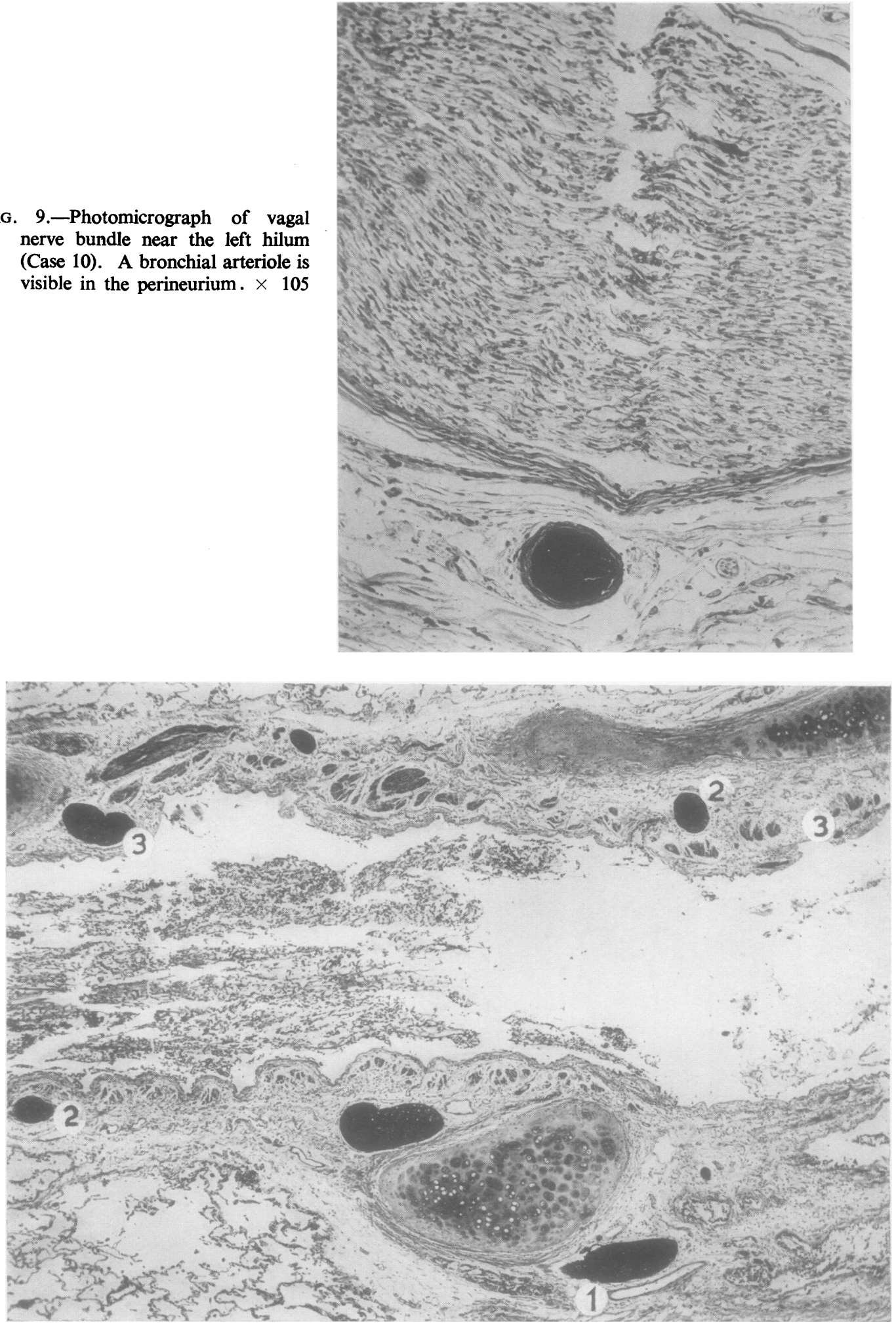

FIG. 10.-Photomicrograph of a longitudinal section of a medium-sized bronchus (Case 9) showing bronchial arterioles in (1) tunica fibrosa, (2) tunica propria, and (3) submucosa. $\times 35$. 


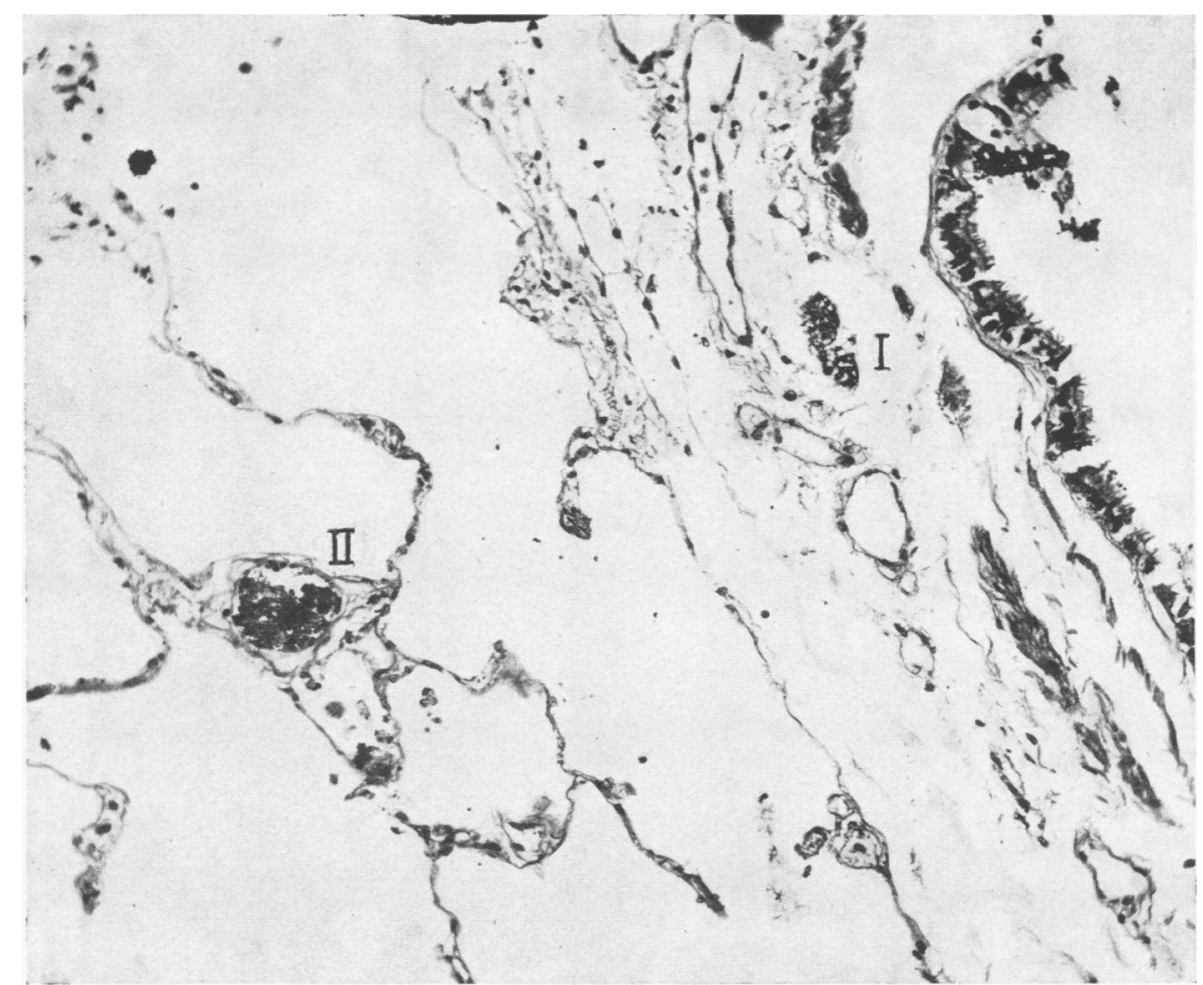

FIG. 11.-Bronchial arteriole (I) near bronchiolus and (II) in supporting tissue of alveolar wall (Case 6). $\times 175$.

\section{Discussion}

In the present study all bronchial arteries appeared to originate from the aorta, but no constancy as to their number or their level of origin was found. This accords with the analysis of Cauldwell, Siekert, Lininger, and Anson (1948), who, in 150 cases, found that the bronchial arteries arose from the aorta in over $90 \%$, that the number of the vessels varied considerably, and that their level of origin in nearly $80 \%$ lay opposite the fifth and sixth dorsal vertebrae.

The present technique revealed a constant radiological pattern in all the 10 cases. This permitted the nomenclature of the bronchial tree to be adapted to the bronchial arterial divisions outlined radiographically. In addition a well-marked annulus surrounding the main bronchi near the hilum and a constant apical pleural branch were recognized.

The presence of bismuth cream in the lumina of the bronchial arteries made the differentiation of these vessels from pulmonary arteries and veins in stained lung sections comparatively simple. The difficulty of differentiating between pulmonary venules and arterioles was well recognized by Brenner (1935). The present method failed to demonstrate pre-capillary anastomoses between the bronchial and pulmonary arteries in normal lungs. The absence of flow from the pulmonary 


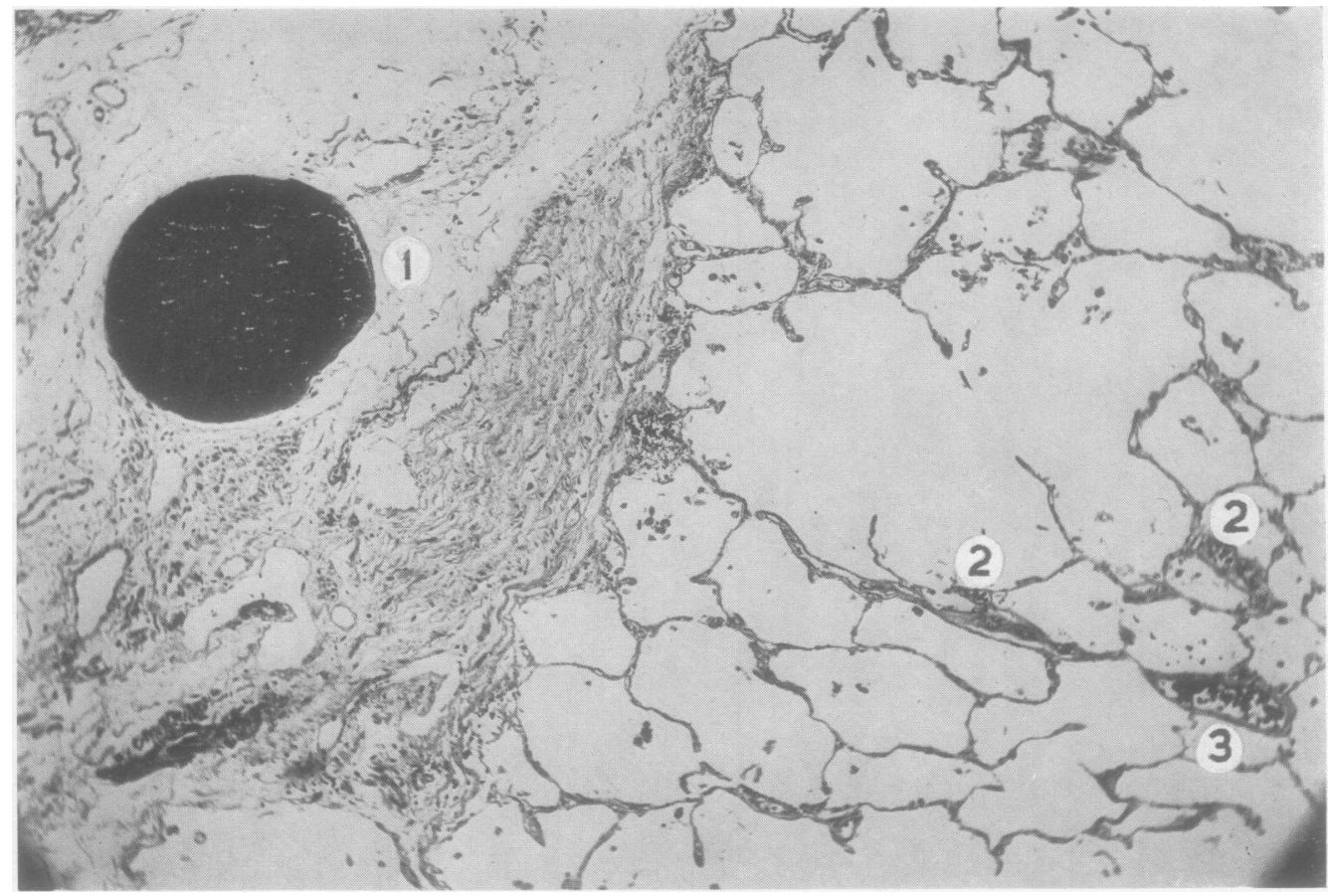

FIG. 12.-Photomicrograph (Case 7) showing bronchial arterioles in (1) interlobular septum and (2) and (3) alveolar septum. $\times 75$.

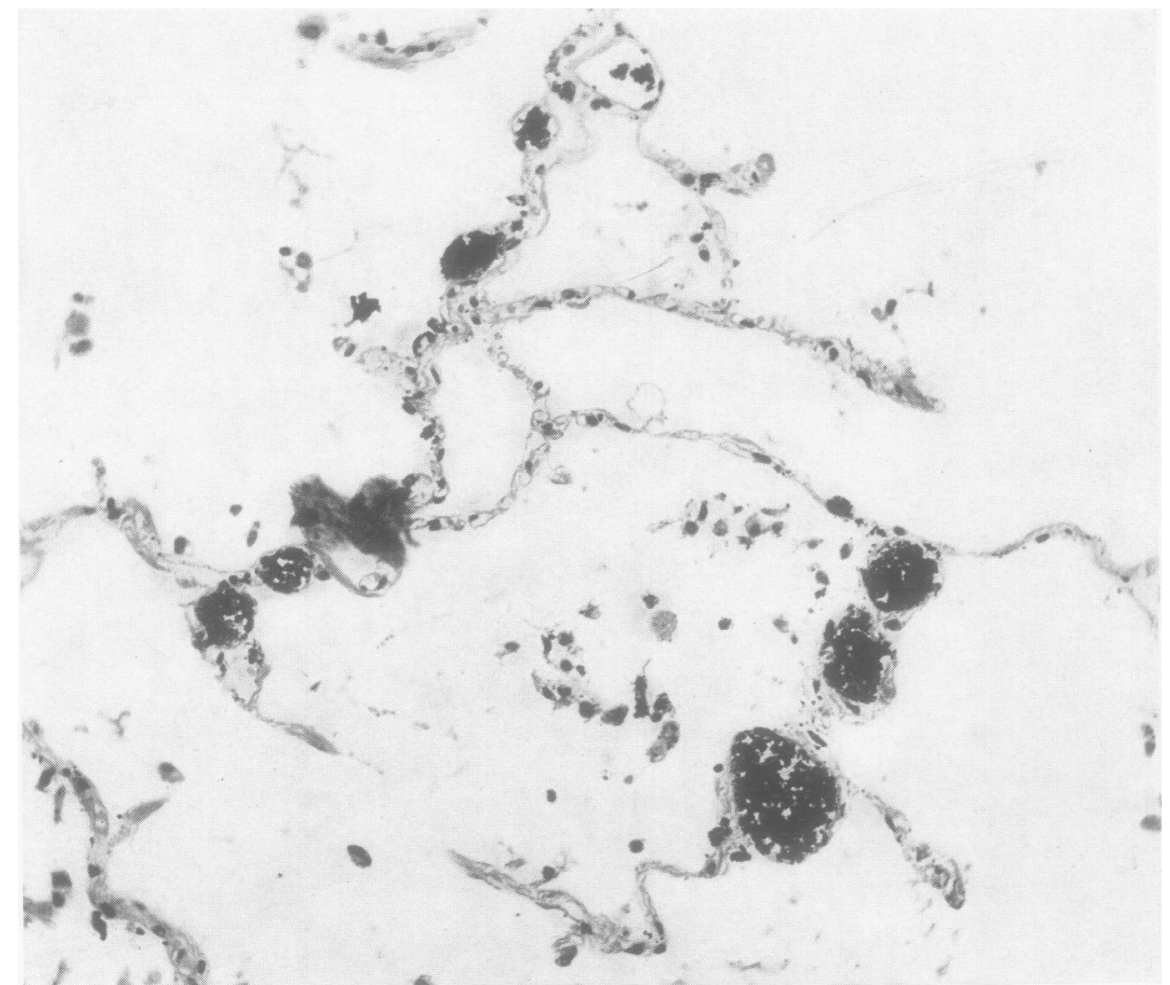

FIG. 13.-Bronchial arterioles in alveolar walls (Case 4). $\times 205$ 


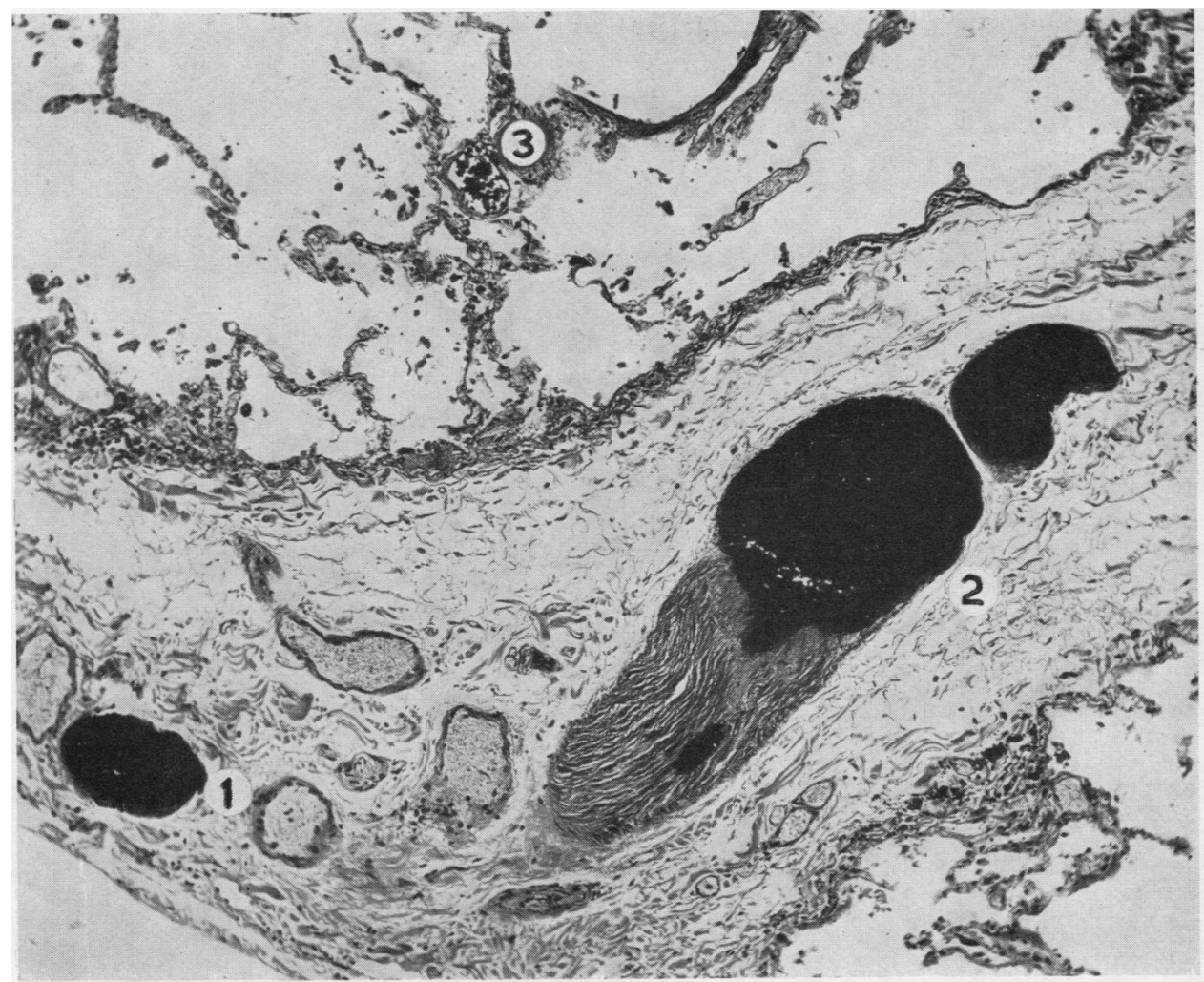

FIG. 14.-Photomicrograph (Case 2) of bronchial arterioles in (1) visceral pleura, (2) interlobular septum, and (3) alveolar wall $\times 88$.

arteries during the preliminary flushing with saline, and the liberal flow from the pulmonary veins, favour Miller's contention that the bronchial capillary bed constitutes an additional source for the pulmonary veins. It would also appear that the bronchial artery bed is independent of the pulmonary artery bed in health, and that pre-capillary communications of the two systems are of pathological significance.

Finally there are numerous bronchial arterioles in the interlobular septa which course along strands of elastic tissue to reach the interstitial tissue of the alveoli. These interlobular arterioles are derived, not from the arteries accompanying the bronchi, but from the pleural arterial system. The arterioles supplying the interstitial tissue of the alveoli could be distinguished from the pulmonary arterioles only by their bismuth-filled lumina. It appears, therefore, that the actual framework of the alveoli has its own systemic arterial blood supply.

\section{SUMMARY}

The bronchial arteries in 10 normal lungs were injected with a radio-opaque medium and studied radiologically and histologically. The territory of the bronchial arteries was found to extend to all lung structures with the exception only of the epithelium of the pulmonary capillaries. No pre-capillary anastomoses between the 
pulmonary and bronchial arteries were found in normal human lungs. A constant radiographic pattern for the bronchial arteries in normal lungs was demonstrated. The histological examination of the intra-pulmonary distribution of the bronchial arteries indicated that the supporting framework of the alveoli receives its own independent arterial blood supply from vessels of the interlobular septa, which reach the air sacs at a direction at right angles to the alveolar ducts.

Some of the material in this study has been incorporated in a thesis by L.C. for the M.D. degree and was accepted by the University of London in December, 1950.

We wish to thank Professor J. McMichael, Dr. J. Crofton, and Dr. C. V. Harrison for placing facilities at our disposal; Dr. B. Lennox for his encouragement ; Dr. J. P. Shillingford, of the London Hospital, for details of the injection medium; the technical staff of the Department of Pathology for cutting and staining the sections ; Miss M. S. McAdam for taking the radiographs ; Mr. C. V. Willmott, F.R.P.S., for the photomicrography, and Mr. K. Moreman, A.R.P.S., for reproducing the radiographs.

\section{REFERENCES}

Berry, J. L. (1935). Quart. J. exp. Physiol., 24, 305.

Brailsford, J. F., and Daly, I. de B. (1931). Proc. roy. Soc. B., 109, 214.

Brenner, O. (1935). Arch. intern. Med., 56, 211, 457, 724, 976, 1189.

Cauldwell, E. W., Siekert, R. G., Lininger, R. E., and Anson, B. J. (1948). Surg. Gynec. Obstet., 86, 395 .

Daly, I. de B. (1935-6). Harvey Lect., 31, 235.

Galen, C. (1562). Tractat. Frobenianae edit., lib. 6, cap. 3. Basel.

- (1854). Oeuvres de Galien, trans. C. Daremberg, vol. 1, p. 385. Paris.

Karsner, H. T, and Ash, J. E. (1912). J. med. Res., 27, 205.

Küttner, C. (1878). Virchows Arch., 73, 476.

Le Fort, L. (1858). " Recherches sur l'Anatomie du Poumon Chez l'Homme." Thèse, Paris.

Luschka, H. von. (1863). Die Anatomie des Menschen, vol. 1: Tübingen.

Mathes, M. E., Holman, E., and Reichert, F. L. (1932). J. thorac. Surg., 1, 339. Reichert, F. L., and Holman, E. (1930). Proc. Soc. exp. Biol., N.Y., $27,278$.

Miller, W. S. (1947). The Lung, 2nd ed., Springfield, Ill.

Reisseisen, F. D., and Soemmering, S. T. von (1808). Uber den Bau Der Lungen. Berlin.

Rindfleisch, E. (1878). Handbuch der Gewebelehre, Leipzig, 5th ed.

Ruysch, F. (1732). Epistola anatomica, Amsterdam.

Shillingford, J. P. (1950). Personal communication.

Thoracic Society (1950). Thorax, 5, 222.

Virchow, R. (1856). Gesammelte, Abhandlungen zur wissenschaftlichen Medicin, p. 285. Frankfurt a.M.

Zuckerkandl, E. (1881). Sb. Akad. Wiss., Wien, Abt. 3, 84, 110.

(1883). Ibid., Abt. 3, 87, 171. 